\title{
El amor interracial como arma de resistencia política en el relato breve «Hora de partir», de César A. Mba Abogo
}

\author{
FRANCISCO FuENTES ANTRÁS \\ Universidad Autrónoma de Madrid \\ fra.fuentes@predoc.uam.es
}

Resumen: El siguiente artículo se centra en el modo en el que Ariadna y Ka, los dos personajes en el relato breve «Hora de Partir» (2007), del escritor ecuatoguineano César A. Mba Abogo, resisten una categorización racial y social dominante mediante la relación de amor que mantienen en España. Para dicho propósito, me sirvo de la ideología del colorblindness, la cual defiende que la igualdad entre categorías grupales se consigue mediante el tratamiento de las personas como seres individuales con características únicas (Rosenthal et al. 2010: 150). La aplicación de esta teoría al análisis de la narración permite resaltar el proceso de individuación que ambos personajes experimentan, basado en el ensalzamiento de su individualidad. De este modo, este artículo defiende que la relación de amor que ambos mantienen funciona como un espacio literario de resistencia a nivel narrativo desde donde los personajes enfrentan los estereotipos sociales, raciales y políticos sobre los que la categorización grupal está conformada.

Palabras clave: literatura de resistencia, categorización grupal, literatura hispano-africana, conflicto intergrupal

The interracial love as a political resistance weapon in the short story «Hora de partir» from César A. Mba Abogo

Summary: The following article explores the ways in which Ariadna and Ka, the main characters in the short story «Hora de Partir» from César A.MbaAbogo, resist a hegemonic racial and socialcategorization through the love relationship they have in Spain. For this purpose, I employ the colorblindness ideology, which argues that treating people as individual beings with unique features fosters the equality among group categories (Rosenthal et al. 2010: 150). By applying this theory to the analysis of the narration, I highlight that the individuation process that both characters undergo is based on the enhancement of their individuality. In this light, the article intends to demonstrate that their love relationship works as a resistance literary space at a narrative level, where both characters find the the social, racial, and political stereotypes upon which group categorization is formed.

Key words: resistance literature, group categorization, Afro-Hispanic literature, intergroup conflict 


\section{Introducción}

T a teoría de identidad social se basa en la premisa de que los individuos construyen sus identidades atendiendo a categorizaciones sociales y de pertenencia al grupo (Tajfel \& Turner, 1986). Dicha categorización ha implicado a menudo la percepción de un individuo como una entidad estrechamente ligada al grupo social, racial, étnico, religioso o sexual al que pertenece, por lo que sus características individuales se ven ignoradas y silenciadas (Dovidio et al., 2009). Por consiguiente, la percepción de una persona como miembro de un grupo tiene un profundo impacto en cómo los seres humanos procesan información sobre otros y actúan hacia los demás (Dovidio et al., 2009). En contraste, en las últimas décadas, se han venido desarrollando diferentes teorías dentro del campo de la psicología social con ánimo de combatir los prejuicios y estereotipos que dividen a miembros pertenecientes a grupos categóricos distintos, y que sostienen que la igualdad y libertad entre personas solo pueden alcanzarse minimizando las distinciones entre miembros de grupos diferentes para así reconocer sus características propias (Rosenthal et al., 2010; Rattan \& Ambady, 2013).

Del mismo modo, estos estudios hacen hincapié en cómo esta supeditación del ser humano al grupo puede llevar a constricciones potenciales en miembros del grupo minoritario, ya que es suficiente para «producir prejuicios tanto a favor de los miembros del grupo propio como de los miembros de grupos ajenos» (Dovidio et al., 2009: 4). Cuando las personas son categorizadas grupalmente, no solo tienden a reducirse las diferencias entre miembros del mismo grupo, sino que también dichos individuos dejan de ser agentes libres. Por el contrario, cuando el individuo se rebela contra su grupo categórico experimenta un proceso denominado individuación, el cual le lleva a la demolición de imposiciones sociales y a la libertad de agencia (Ross,1920: 469).

Este artículo se centra en el relato breve «Hora de Partir», de César A. Mba Abogo, incluido en su libro de relatos y poemas El Porteador de Marlow: canción negra sin color (2007), con el objetivo de analizar cómo los protagonistas luchan por su libertad de agencia al combatirla categorización grupal y retar estereotipos y distinciones grupales emprendiendo una relación amorosa interracial. En efecto, defiendo que es por medio de establecer una relación íntima con un miembro de otro grupo que Ka, un negro de origen africano, 
y Ariadna, una blanca europea, deslegitiman el conflicto intergrupal, fomentan su individualidad, y promueven un diálogo transcultural. Como consecuencia, la relación amorosa que ambos emprenden en la narración funciona como espacio literario de resistencia, concepto inspirado por la noción de «espacios activos» de Doreen Massey, y que se define como un espacio en el cual un individuo adquiere libertad de agencia y desde el que puede operar (Massey,1995: 54). El espacio intersticial que la relación amorosa representa a nivel narrativo permite a los protagonistas adquirir una voz crítica y encontrar un modo efectivo de resistir las constricciones sociales y raciales impuestas por la categorización grupal.

Asimismo, numerosas investigaciones dentro de la psicología social han abordado los efectos negativos de la categorización en grupos (Tajfel and Turner, 1986; Dovidio et al., 2009; Pehrson \& Green, 2010) al señalar dicha categorización como la causa principal del conflicto y el prejuicio intergrupal. En este sentido, dado que la categorización grupal ejerce de base en la construcción de un pensamiento sesgado y actitudes discriminatorias entre miembros de grupos ajenos (Tajfel \& Turner, 1986), expertos en el campo de la psicología social han tratado este proceso como un punto de partida en lo que respecta a la mejora de las relaciones entre grupos.

Más específicamente, académicos como Aneeta Rattan y Nalini Ambady han profundizado en el estudio de las ideologías intergrupales (con mayor enfoque en el multiculturalismo y en el color blindness) con el fin de subrayar la importancia de la diversidad ideológica en lo que respecta a la investigación psicológica de las relaciones intergrupales y a combatir el pensamiento intergrupal sesgado. Mientras la ideología del multiculturalismo aboga por la pertenencia al grupo y la necesidad de valorar dicha pertenencia y las diferencias entre grupos para alcanzar la igualdad y la diversidad entre personas (Rattan \& Ambady, 2013: 12), la ideología del color blindness defiende, por el contrario, que la igualdad entre grupos se logra minando las distinciones entre los mismos y reconociendo a las personas como individuos únicos y no como entidades apegadas a una comunidad (Rattan \& Ambady, 2013: 12; Rosenthalet al., 2010: 150).

Debido a que, a mi juicio, la libertad de agencia de los protagonistas se produce como resultado de desafiar las distinciones existentes entre sus categorías grupales, en este análisis de «Hora de Partir», me sirvo de la ideología 
del color blindness para resaltar el proceso de individuación que ambos personajes experimentan con el objetivo de enfrentar los prejuicios generados por el pensamiento sesgado del endogrupo hacia miembros de grupos ajenos. Ya que por individuación entiendo el proceso que pulveriza las ataduras sociales de un individuo en favor de su libertad de agencia (Ross,1920: 469), tanto Ka como Ariadna deben liberarse de las constricciones ideológicas que sus grupos categóricos les imponen para deslegitimizar los conflictos intergrupales generados por prejuicios y disparidades que impiden su autonomía e independencia.

\section{Fuentes de conflicto intergrupal en «Hora de partir»}

Las causas que generan los conflictos intergrupales han sido ampliamente investigadas en una variedad de disciplinas. Mientras que algunos académicos apuntan a las diferencias religiosas y étnicas como una excusa para crear conflicto donde el objetivo real es la obtención de recursos tangibles y poder, otros achacan la motivación del conflicto intergrupal a las diferencias ideológicas y de valores entre grupos (Neuberg et al., 2013). De manera importante, este tipo de conflicto entre miembros de grupos distintos se ha representado a través de violencia individual, grupal, o actos simbólicos de agresión (Neuberg et al., 2013).

A mi juicio, los conflictos que sendos personajes deben enfrentar son el resultado de prejuicios y estereotipos principalmente entorno a las dos fuentes centrales de distinción social en la narración: la nacionalidad y la raza. Para empezar, cabe resaltar el nacionalismo como causa de conflicto intergrupal ya que, desde el inicio de la narración, Ka es representado como un ciudadano de segunda en Europa debido en parte a su condición de inmigrante africano. Tal y como afirman Pehrson y Green, la hostilidad hacia el inmigrante es alentada por la sospecha hacia grupos étnicos o religiosos, que son vistos como una amenaza para la cohesión nacional y la seguridad que el espacio nacional dice proporcionar (2010: 696). En efecto, las actitudes negativas hacia el inmigrante han sido extensamente investigadas, enfocándose en la forma en que los grupos nacionales construyen límites y aplicando con éxito teorías relativas a la psicología social sobre el prejuicio y el conflicto intergrupal al fenómeno de la inmigración (Pehrson \& T. Green, 2010; Rodríguez 
et al., 2013; Sabucedo y Fernández, 1998). Durante todo el relato Ka es presentado como un ser externo que ha de realizar un esfuerzo por adentrarse en el nuevo mundo en el que desea estar. Dicho espacio está representado por la figura de Ariadna, la protagonista nativa, por lo que la relación amorosa entre ambos podría analizarse como un intento de convergencia entre el espacio nativo y el extranjero, donde ambos personajes, en representación de sus espacios, cruzan las barreras categóricas entre el «nosotros»y el «ellos» que el endogrupo impone tanto a sus propios miembros como a individuos externos (Rodríguez et al., 2013).

De esta manera, en el primer párrafo de «Hora de Partir», la voz narrativa revela que, una vez en Europa, Ka debe acortar su nombre africano, Karaevini Ekang, «como una fórmula ritual para acortar la historia (tan indecisa, con tantas ansias, sin límites) de su vida» (Abogo, 2007:63). En otras palabras, puede afirmarse que, con motivo de ser más comprendido por los nativos de su nueva tierra y encajar en los parámetros nacionales europeos, Ka se ve obligado a modificar su historia, lo que le sitúa desde un primer momento en una posición desaventajada como ciudadano al verse en la obligación de renunciar a parte de su identidad si quiere adaptarse más fácilmente a la nueva tierra.

Esta condición de ciudadano de segunda es igualmente acentuada cuando el narrador menciona cómo las patrullas policiales preguntan a menudo a Ka por sus papeles en plena calle, o su miedo justificado de «no conseguir nunca un trabajo que se ajustara a sus capacidades» (2007: 74). De hecho, las actitudes intolerantes y de desconfianza hacia el inmigrante se basan en un miedo a la contaminación cultural que suele acompañar a la evaluación positiva de la nación y su reforzamiento (Rodríguez et al., 2013: 39). Como consecuencia, Ka tiene la sensación de ser tratado como un «agente patógeno» (Abogo,2007: 170) que se ve, tal y como lo expresa la voz narrativa, «arrastrándose por las calles de Europa como una cosa desvalida sin órganos, envejeciendo delante de histerectomizadas expresiones...» (2007: 170). La negatividad del epíteto "patógeno» (definiéndole como algo que causa enfermedad) y la comparación del protagonista con «una cosa sin órganos» (170) evidencia la deshumanización que Ka experimenta en Europa y la actitud de desconfianza e indiferencia que los nativos le muestran debido a su condición de inmigrante africano. 
En segundo lugar, la raza constituye la otra fuente de conflicto intergrupal que promueve la categorización en grupo e impide la libertad de agencia de los protagonistas. La diferencia racial y los prejuicios derivados de esta categorización suponen obstáculos decisivos que van adquiriendo fuerza a medida que avanza la narración hasta finalmente demoler la relación entre los personajes, tal y como se puede deducir del siguiente extracto:

Pero, finalmente, el timbre del mundo cruel y cruento había encontrado un hueco por el que penetrar y apagar la utopía de los cronopios. Y el timbre trajo a las patrullas policiales que le preguntaban, en plena calle, por los papeles de $\mathrm{Ka}$, el miedo justificado de Ka de no conseguir nunca un trabajo que se ajustara a sus capacidades, el miedo y la vergüenza de Ka de tener que demostrar siempre de buenas a primeras que él era «un negro diferente», la rabia de Ariadna de tener que enumerar los méritos intelectuales de Ka de buenas a primeras para que su relación con "el chico negro" no fuera vista como una aberración (Abogo,2007: 74).

La voz narrativa no solo resalta la condición de inmigrante, sino también la raza, como la fuente principal de conflicto que dificulta la relación amorosa de los personajes. Mediante el empleo de las palabras «negro diferente» por parte del entorno de Ariadna para aludir a Ka, o el hecho de que se pongan en duda los méritos intelectuales del mismo, este extracto evidencia la categoría de ciudadano de segunda que el personaje adquiere, no solo debido a su condición de extranjero, sino también a su color de piel. De hecho, la alusión al color es constante a lo largo del relato, a través del apodo que Ariadna recibe de Ka, «la Nefertiti Blanca», o la referencia a la película «gato Negro, gato Blanco» (67). Otros ejemplos en los que el color adquiere protagonismo son esos momentos en los que el narrador se refiere explícitamente al mismo como causa de conflicto intergrupal y obstáculo en la relación de amor que mantienen los personajes. Cuando los protagonistas se disponen a romper su vínculo, el narrador menciona a Ka de nuevo «viajando entre las tinieblas de su memoria negra» $(73)^{1}$, pese a que «durante meses ambos habían jugado a despigmentarse» (74). Asimismo, puede apreciarse el temor de $\mathrm{Ka}$ a ser rechazado en su primera cita con Ariadna debido a su color de piel:

1 Parece ser un guiño del autor a la novela homónima de Donato Ndongo-Bidyogo, obra seminal de la literatura ecuatoguineana. 
Un árbol cayó sobre Ka, las raíces del rechazo pigmentado se trenzaron en su ánimo diapositivando una y otra vez la misma imagen. Allí estaba otra vez ese miedo. El miedo crudo, cortante, vertical, que ya tanto conocía pero que se presentaba agudo y doloroso, como si jamás se hubiera materializado esa triste realidad que enjarciaba cada rechazo, cada portazo, cada mirada vacía, sorda y punzante que se clavaba en su piel cada vez que se presentaba a un anuncio. [...] Pero su cuerpo le contradecía. Deseaba ir a ver la casa y de paso decapitar la sensación de apestado humano que los rechazos pigmentados habían hecho nacer en él (Abogo, 2007: 66-67).

Este extracto muestra el miedo que siente el protagonista a la posibilidad de recibir un rechazo por parte de Ariadna a causa de su color de piel, y la frase «rechazo pigmentado» evidencia esa relación existente entre el miedo a no ser aceptado y su raza. No obstante, Ka reúne finalmente el valor para enfrentar la sensación de apestado humano que los «rechazos pigmentados» le causan y acudir a su cita con Ariadna.

Por lo tanto, el texto alude a un conflicto intergrupal basado en distinciones y prejuicios sociales y raciales, lo cual provee a la historia de dramatismo y presenta un retrato realista de estos conflictos. La condición de inmigrante y el color negro de Ka constituyen las fuentes de conflicto intergrupal sobre las que se centra la narración, puesto que ambos personajes han de enfrentar los prejuicios basados en dichas fuentes para llevar a cabo su unión. El ser miembros de grupos categóricos distintos (nativa/inmigrante, blanca/ negro), y la tendencia de los grupos a deshumanizar a miembros de los exogrupos constituyen obstáculos para poder desarrollarse como agentes libres, y no como individuos apegados a una determinada comunidad.

\section{Modos de resistencia contra la categorización grupal en «Hora de partir»}

Sin embargo, cabe resaltar que las dificultades anteriormente señaladas son contratacadas por la relación intergrupal que los personajes establecen y la consecuente interacción de sus mundos, lo cual favorece la minimización de las fronteras categóricas y una coexistencia que ensalza sus características comunes. En primer lugar, postulo que la voz narrativa hace referencia a elementos transculturales que promueven la conexión entre diferentes, y que esta amalgama es lograda a través de la evocación constante de un ambiente 
transcultural. En el relato «Hora de Partir», elementos africanos y europeos se enlazan evocando un ambiente intercontinental que enmarca la narración. Por ejemplo, la contundencia de los latidos del corazón de Ka cuando ve a Ariadna por primera vez es comparada con «una batucada» (2007: 64), siendo esta una clara referencia a la música africana. Asimismo, Ka comparte con su amante su conocimiento sobre autores africanos y escuchan juntos «los sanadores espirituales de llaga africana» (69). De esta forma, encontramos más elementos del imaginario africano, como los que surgen de la curiosidad de Ariadna hacia la cultura de Ka: «Ariadna no paró de hacerle preguntas a $\mathrm{Ka}$, con los ojos brillantes como un felino agazapado tras un arbusto en una noche negra de mayombe, sobre África» (68). De igual manera, el narrador hace referencia a elementos europeos, tales como «guitarra española» (65) o «decorado de una película independiente» (65) para describir la visión de Ka sobre su barrio, los versos de Tennyson que Ariadna lee en voz alta cuando está en casa, o el collage de Hundertwaser sobre su cabecero de la cama. En ocasiones, la voz narrativa trasciende la frontera geográfica al referirse explícitamente a una universalidad:

Un argentino, con una voz rasposa y profunda, guitarra española en mano, [...] a ritmo de un tango de Obango, al ilustre Sarava de forma inadvertidamente feliz. Unos camareros fácilmente irritables entraban y salían del local con unas coquetas bandejas. Unos niños magrebíes jugaban al fútbol en la plaza. Un grupo de Muyahidines formaban un círculo en uno de los extremos de la plaza, charlaban amigablemente, y de vez en cuando el suave viento aterciopelado sacudía sus barbas exuberantes. Velos islámicos. Turbantes sijs. Gente paseando abrazada. Balcones adornados con macetas y sabanas que gritaban su repulsa a la guerra de Iraq. [...] Con los ojos puestos en muchas partes del planeta... Todo parecía tener una belleza frágil. Indescriptible (2007: 65).

En este extracto el barrio de Ka se describe en términos transculturales mediante el empleo de elementos que aluden a culturas dispares, como por ejemplo «guitarra española», «turbantes», «velos», «tango» o «niños magrebíes». Asimismo, se asocia este escenario de diversidad a un ambiente de armonía y coexistencia pacífica al mencionar cómo los niños magrebíes juegan al fútbol, al grupo de Muyahidines charlando amigablemente, o a gente abrazada. De manera importante, la representación de un contexto transcultural posible ejerce como marco narrativo e impulsa la relación intergrupal 
entre los personajes. De hecho, la pareja resiste la categorización grupal a través de su coexistencia pacífica, la cual establece lazos intergrupales y los libera al mismo tiempo de las constricciones categóricas.

Este último punto nos conduce al otro modo en el que los prejuicios intergrupales son combatidos y que se funda en el interés que ambos personajes mantienen por conocer la cultura del contrario y por intimar. La comunión entre Ka y Ariadna está basada en este interés que muestran el uno por el otro pese a las dificultades, y que puede percibirse en sus continuas preguntas sobre el trabajo, los familiares y las experiencias pasadas que se realizan entre sí. Este interés genuino entre miembros de grupos distintos está muy presente en el relato «Hora de Partir».Según Ross, «la diversificación que tiene lugar en cada cultura como consecuencia de la penetración de elementos de otras culturas amplía el abanico de elección individual» (1920: 479). Así, puede afirmarse que las relaciones intergrupales, con su consecuente interculturalidad, afectan de forma positiva al individuo en tanto que este se ve empoderado, contrariamente al efecto que en él causan los grupos categóricos.

En este sentido, puede reconocerse la intención del narrador de crear fuertes lazos emocionales entre los personajes a lo largo del relato, y que fomentan enormemente el refuerzo de la comunión entre ambos. Desde el inicio de la narración, el empleo de lenguaje cargado de emotividad como «el limo amargo de la despedida» (63), ojos «humedecidos y a puntito de llorar» (63) o «espirales de tristeza» (64) contribuye a la evocación de un ambiente íntimo y emocional marcado por los sentimientos de ambos personajes. El que la narración abra con la despedida de la pareja, ya habiendo terminado la relación, y con el llanto de Ka, supone también aportar carga emocional a la historia y resalta el potente vínculo amoroso que la pareja interracial posee. De hecho, en las primeras líneas del relato, el narrador se adentra en las emociones de Ka, quedando su intimidad descubierta al revelar cómo sus ojos se humedecen a causa de la tristeza que la hora de partir, o su separación de Ariadna, le produce. Del mismo modo, aunque la narración haga hincapié en el personaje masculino, el narrador también ofrece en las primeras líneas un retrato de Ariadna en un estado de aflicción, al aludir a sus «pupilas dilatadas»(64) o la forma en la que acaricia a Ka y deja su mano «perdurarse»(64) en la boca de su amado. 
Por lo tanto, la relación romántica entre Ka y Ariadna y el esfuerzo que ambos hacen para mantenerla a pesar de los múltiples obstáculos implica un interés real en los pensamientos y el modo de vida del otro ya que, mientras Ka está «deseoso de leer todos los espacios de la vida de Ariadna» (67), las preguntas de ella sobre África son constantes. Este interés que ambos tienen el uno por el otro es evidente desde que se ven por primera vez:

La primera vez que Ka vio a Ariadna fue en la terraza de un café que languidecía a los pies de un horrible edificio que hacía de Museo de Arte Contemporáneo. [...] El aire tenía un aroma fresco y húmedo. La tarde aflojaba, la luz se adormecía bajo los párpados del sol. El sol, sin apenas hojas, languidecía. Los contornos de las cosas se desvanecían. [...] En aquel instante, mientras la descifraba, Ka se preguntó cómo es que aquella chica no estaba maravillada de sí misma (Abogo, 2007: 64-65).

La voz narrativa adopta la perspectiva de Ka para describir el momento en el que se encuentra con Ariadna. La alusión a la protagonista como un ser cautivador contrasta con la descripción del ambiente donde se produce el encuentro, el cual es referido en términos negativos como una terraza que languidece junto a un edificio horrible o un sol apagado. La referencia al aire fresco y húmedo rompe con la sordidez del ambiente y parece ejercer como un recurso poético que anunciala llegada de Ariadna a la vida de Ka. En ese mismo instante, el narrador menciona como Ka comienza a descifrar a su futura pareja, y es este verbo (descifrar) el que, a mi juicio, porta una gran carga semántica en la narración al funcionar como una doble alusión: por un lado, resalta el interés que el protagonista tiene por conocerla, y por otro, anuncia la predisposición del personaje a adentrase en lo más hondo de su amada a pesar de los obstáculos.

Por consiguiente, tanto el deseo de Ka de descifrar a Ariadna como la admiración por su belleza evidencian la curiosidad que tiene con respecto a esta chica europea. En este sentido, la frase «los contornos de las cosas se desvanecían» (64) constituye una reveladora metáfora de como ambos personajes cruzan límites de categorización (basados en pensamientos binarios de blanco/negro, nativo/inmigrante) para comenzar una relación. Este acto de resistencia hacia la categorización grupal culmina cuando deciden vivir juntos y «sus formas se [juntan] y se [dispersan] como rayos de sol» (67). Las imágenes construidas en el relato a partir del difuminado de contor- 
nos geométricos y la comparación de los personajes con sustancias carentes de forma (como los rayos de sol) hacen referencia a la ruptura de fronteras categóricas por parte de los personajes. Al despojarse figurativamente de sus cuerpos, Ka y Ariadna retan, mediante su unión, las demarcaciones que separan al endogrupo de miembros de otros grupos o, más específicamente, las barreras que los nacionales edifican entre ellos mismos y los inmigrantes (Pehrson \& Green,2010: 696).

De hecho, cabe resaltar que esta unión física y espiritual entre los personajes constituye una manera de rebelarse contra la homogamia, la práctica que consiste en la unión sexual y romántica de personas basada en similitudes raciales, étnicas, ideológicas y socioeconómicas (Killian,2013: 125). Killian defiende que elegir casarse con una pareja de una raza o etnia distinta puede acarrear conflictos sociales y psicológicos serios con amigos y familiares, y que es mediante la violación de las expectativas sociales de la homogamia, la conformidad social y la segregación, cuando las parejas interraciales representan una resistencia hacia instituciones e ideologías sociales (2013: 190).

Por tanto, el sexo y el romance interracial pueden ser entendido como un comportamiento desviado y una forma de resistencia al pensamiento supremacista homógamo. De hecho, las escenas sexuales entre Ka y Ariadna descritas en el relato juegan igualmente un papel muy importante en la consumación de su unión y evocan la rotura de las limitaciones raciales y sociales: «ambos querían morir el uno dentro del otro, convertirse en substancias inanimadas, recorrer toda la gama de la existencia pre-orgánica y post-orgánica, y renacer una y otra vez, un millón de veces, ad infinitium, para volver a amarse» (Abogo,2007: 67).

Su conversión en «substancias inanimadas» alude también a su liberación de las rígidas líneas divisorias que encarcelan a ambos personajes en parámetros de categorización racial y social evocados a través de «nomenclaturas segregacionistas» y «los temores geométricos». Ka y Ariadna viajan hacia «la gama de la existencia», es decir, la amalgama de colores. Así, su fusión les transporta a «un espacio nuevo» (68) donde las distinciones grupales se minimizan en nombre de su amor mediante la construcción de una «burbuja particular» en el interior de «la gran Burbuja en la que los fanatismos de unos [...] abren las puertas del infierno de otros» (74). Esta metáfora hace referencia a los prejuicios que tienen que enfrentar como resultado de ca- 
tegorizaciones grupales, y a la necesidad de construir un espacio privado donde ejercer su amor libremente.

De este modo, las relaciones sexuales que mantienen también ejercen como un comportamiento de resistencia frente al pensamiento homógamo, y es mediante la práctica sexual que ambos personajes logran su comunión total:

A pesar del ensueño su cuerpo había recibido el mensaje y respondía con temblores de deseo, intensos, envolventes. Su cuerpo se sublevó. Se ofrecía latente a la espera de un dedo que lo incitara. Empezaron a besarse y a susurrarse frases cardíacas. Sin prisa fueron perdiendo la noción de todo cuanto les rodeaba, tensando sus cuerpos para encadenarse hasta el infinito (Abogo, 2007: 69-70).

Términos como «envolvente», «besarse»o «susurrarse»aluden al acto de acercarse física y espiritualmente. En este extracto, la interconexión es representada como infinita, más allá de los límites del tiempo y la existencia, evidenciando así sus encuentros sexuales como el acto de máxima intimidad entre miembros de grupos categóricos distintos.

\section{3. «Hora de partir» como narrativa de resistencia política}

No obstante, habiendo reconocido el acto de resistencia que ambos personajes llevan a cabo y la consecuente erosión de las fronteras categóricas, es necesario destacar que el relato aporta un retrato realista al describir la comunión completa entre miembros de grupos diferentes como un proceso arduo que, en este caso, permanece inacabado al final de la historia. En «Hora de Partir»,el triunfo de los personajes en lo que concierne a su unión no es total, ya que a pesar de que tanto Ka como Ariadna llevan a cabo una relación intergrupal y logran encarnar individuos resistentes al fomentar la coexistencia entre diferentes, su deseo de prolongar su relación se ve interrumpido finalmente debido a los obstáculos. Como afirma Julia Borst en su análisis de «Hora de Partir», «la pareja mixta como tema recurrente de la coexistencia transcultural [...] es lentamente deconstruido a lo largo del relato» (Borst, 2017: 43). Borst señala un racismo firmemente enraizado en la sociedad como causa de los obstáculos con los que Ka y Ariadna tienen que lidiar para derrotar las diferencias, y entiende el legado del colonialismo y 
los prejuicios como las principales consecuencias de este racismo (2017: 44), tal y como muestra el siguiente extracto:

Su voz, amarga y salvaje, era como un grito triste y frío, aunque hablaba en un tono bajo.

-No podemos seguir así, eso no puede seguir así. Te niegas a enterrar a tus muertos, quieres vivir bajo su sombra, quieres destruirte.

Las palabras de Ariadna rebotaron contra las paredes, pintadas de azul cielo (Abogo, 2007: 71).

En efecto, Ariadna culpa a Ka por no ser capaz de distanciarse del grupo categórico marcado por su raza. Al mismo tiempo, Ka culpa a Ariadna de tratarlo como uno de esos objetos africanos de curiosidad y deseo de los que ella pretende absorber todo el conocimiento (Borst, 2017:44), así como el narrador deja entrever en frases del estilo «Ariadna no paró de hacerle preguntas a Ka [...] sobre África» (Abogo,2007: 68). Como resultado, tal y como concluye Borst en su análisis del relato, al final de la historia ambos protagonistas se dan cuenta de que «los estigmas de la historia son más poderosos que su amor, y sus esfuerzos por despigmentarse son en vano» (2007: 44).

Sin embargo, pese a ser consciente del fracaso de la relación que los personajes mantienen, he querido enfocar mi análisis en la representación de la heroicidad que, a mi juicio, Ka y Ariadna encarnan en la narración. La proeza de los personajes viene marcada por su osadía a la hora de cruzar límites categóricos basados en conflictos intergrupales en torno a la categorización racial y nacional, así como por la lucha que ambos emprenden a favor de su libertad de agencia. De este modo, postulo la importancia de entender la relación de amor que construyen como un espacio literario de resistencia donde los personajes logran encontrar cobijo y resistir contra las imposiciones de grupos categóricos. Basándome en la idea de Ian Chambers de que escribir es viajar, entrar a un espacio, una zona, un territorio, y que abre un espacio que invita al movimiento (Chambers, 1994: 10), entiendo que la relación íntima entre Ka y Ariadna les aporta movimiento, en tanto que les permite cruzar líneas categóricas y esquivar la fijeza que la categorización grupal impone a las personas. Ellos «viajan hacia la gama de su existencia» (Abogo,2007: 67), es decir, la coexistencia de diferentes, 
lo que los transporta a ese «espacio nuevo» (2007: 68) que el narrador menciona y que viene marcado por la ausencia de fanatismos de índole racial y nacional.

Para concluir, a pesar de la imposibilidad de alcanzar una comunión real y completa (escenificada en la narración mediante la ruptura de $\mathrm{Ka}$ y Ariadna a causa de los continuos obstáculos), el relato «Hora de Partir» retrata dos individuos que resisten la categorización grupal al lograr un acercamiento entre ambos. Si bien pertenecen a una raza e identidad nacional distinta, la amalgama intercultural como marco narrativo, el interés que muestran el uno por el otro, y su deseo de crear lazos entre ellos constituyen herramientas efectivas para combatir estereotipos y un modo exitoso de erosionar el conflicto intergrupal a nivel narrativo. Estas armas de resistencia política surgen a raíz de la relación de amor interracial que mantienen, y que funciona como un espacio literario de resistencia donde ambos, mediante un proceso de individuación, adquieren una voz propia desligada del pensamiento sesgado que el grupo categórico impone en los miembros del endogrupo. En este sentido, el relato deslegitima la categorización grupal al mostrar como la penetración en grupos teóricamente contrarios amplía la libertad de agencia de los protagonistas y los empodera como individuos, ya que su interés mutuo, basado en una atracción cultural y sexual, de amor, y amistad, debilita las barreras categóricas a favor de una posible coexistencia entre diferentes.

\section{Referencias bibliográficas}

Aвogo, C. M. A (2007). «Hora de Partir».El porteador de Marlow. Canción negra sin color. Madrid: Sial / Casa de África, pp. 63-74.

Borst, J (2017). «To Be Black in a White Country- On the Ambivalence of the Diasporic Experience in César A. MbaAbogo's El porteador de Marlow. Canción negra sin color (2007)». Research in African Literatures, vol. 48, no. 3,pp. 33-54. JSTOR, DOI: 10.2979/reseafrilite.48.3.06 [9/11/2019].

Chambers, I (1994).Migrancy, culture, identity. Londres y Nueva York: Routledge. 
Dovidio, John F., Samuel L. Gaertner y Tamar Saguy (2009). «Commonality and the complexity of 'we': Social attitudes and social change».Personality and Social Psychology Review, vol. 13, pp. 3-20. Sage Journals, doi. org/10.1177/1088868308326751 [10/11/2019].

Killian, Kylie D. (2013). Interracial Couples, Intimacy \& Therapy. Crossing Racial Borders. Nueva York: Columbia University Press.

MASSEY, D (1995). «The conceptualization of place». En Doreen Massey y Pat Jess (Eds.) A Place in the World?. Oxford: Oxford University Press, pp. 45-58.

NeuberG, S. L., Caroline M. Warner, Stephen A. Mistler, Eric D. Hill, Anna Berlin, Jordan D. Johnson, Gabrielle Filip-Crawford, Roger E. Millsap, George Thomas, Michael Winkelman, Benjamin J. Broome, Thomas J. Taylor, Juliane Schober (2013). «Religious Infusion and Intergroup Conflict: Results from the Global Group Relations Project». Psychological Science, vol. 25, no.1,pp. 198-206.ResearchGate, https:// doi. org/10.1177/0956797613504303 [15/11/2019].

Pehrson, S. y Eva G.T. Green (2010).«Who We Are and Who Can Join Us: National Identity Content and Entry Criteria for New Immigrants».Journal of Social Issues, vol. 66, no. 4, 2010, pp. 695-716. SPSSI, https://doi. org/10.1111/j.1540-4560.2010.01671.x [11/11/2019].

RATTAN, A., yNaliniAmbady. (2013). «Diversity ideologies and intergroup relations: An examination of colorblindness and multiculturalism». European Journal of Social Psychology, vol 43, no.1, pp. 12-21. Wiley Online Library, doi.org/10.1002/ejsp.1892 [10/11/2019].

Rodríguez, A. Verónica Betancor y Eva Ariño (2013). «Identidad nacional y prejuicio. ¿Está el nacionalismo asociado a la xenofobia? / National identity and prejudice: Isnationalismassociated with xenophobia?». Estudios de Psicología, vol. 34, no. 1, pp. 37-47. Research Gate, DOI: 10.1174/021093913805403138 [11/11/2019].

Rosenthal, L., S.R. Levy, y Ian Moss (2010). «The colorblind, multicultural, and policultural ideological approaches to improving intergroup attitudes and relations».Social Issues and Policy Review, vol. 4,pp. 215-246. SPSSI,doi.org/10.1111/j.1751-2409.2010.01022.x [10/11/2019]. 
Ross, Edward A (1920). «Individuation». American Journal of Sociology, vol. 25, no. 4, pp. 469-479. https://www.journals.uchicago.edu/doi/ abs/10.1086/213055 [02/11/2019].

SAbucedo, J.M. y C. Fernández (1998). Nacionalismos e Ideología». Psicología Política, no.17, pp. 7-19. https://www.uv.es/garzon/psicologia\%20politica/N17-1.pdf[12/11/2019].

TAJFel, H. \& Turner, J.C (1986).«The Social Identity Theory of Intergroup Behavior». En S. Worchel and W.G. Austin (Eds.) Psychology of Intergroup Relations, Chicago: Nelson-Hall, pp. 7-24. 